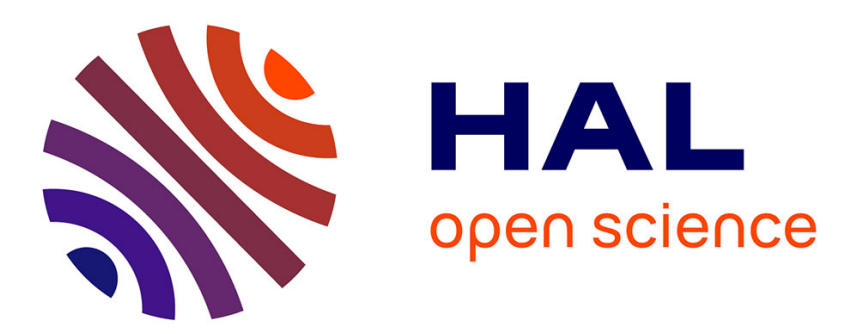

\title{
Transient optoelastography in optically diffusive media
} Emmanuel Bossy, Arik Funke, Khalid Daoudi, Albert-Claude Boccara, Mickaël Tanter, Mathias Fink

\section{To cite this version:}

Emmanuel Bossy, Arik Funke, Khalid Daoudi, Albert-Claude Boccara, Mickaël Tanter, et al.. Transient optoelastography in optically diffusive media. Applied Physics Letters, 2007, 90 (17), pp.174111. 10.1063/1.2733604. hal-02481840

\section{HAL Id: hal-02481840 \\ https://hal.science/hal-02481840}

Submitted on 17 Feb 2020

HAL is a multi-disciplinary open access archive for the deposit and dissemination of scientific research documents, whether they are published or not. The documents may come from teaching and research institutions in France or abroad, or from public or private research centers.
L'archive ouverte pluridisciplinaire HAL, est destinée au dépôt et à la diffusion de documents scientifiques de niveau recherche, publiés ou non, émanant des établissements d'enseignement et de recherche français ou étrangers, des laboratoires publics ou privés. 


\section{Transient optoelastography in optically diffusive media}

Cite as: Appl. Phys. Lett. 90, 174111 (2007); https://doi.org/10.1063/1.2733604

Submitted: 05 February 2007 . Accepted: 31 March 2007 . Published Online: 26 April 2007

Emmanuel Bossy, Arik R. Funke, Khalid Daoudi, Albert-Claude Boccara, Mickael Tanter, and Mathias Fink

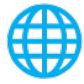

\section{ARTICLES YOU MAY BE INTERESTED IN}

Detection and discrimination of optical absorption and shear stiffness at depth in tissuemimicking phantoms by transient optoelastography

Applied Physics Letters 94, 154103 (2009); https://doi.org/10.1063/1.3120552

Ultrasound-modulated optical computed tomography of biological tissues

Applied Physics Letters 84, 1597 (2004); https://doi.org/10.1063/1.1651330

Optical detection of ultrasound at a distance using a confocal Fabry-Perot interferometer Applied Physics Letters 47, 14 (1985); https://doi.org/10.1063/1.96411

Lock-in Amplifiers Find out more today

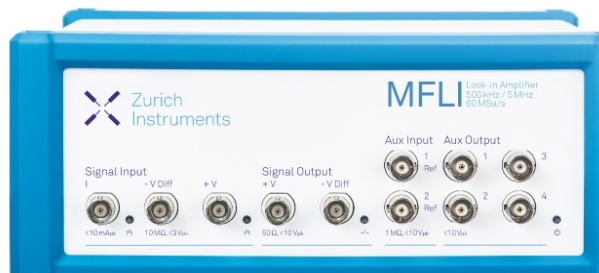

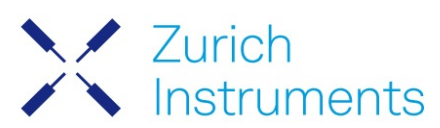




\title{
Transient optoelastography in optically diffusive media
}

\author{
Emmanuel Bossy, ${ }^{\text {a) }}$ Arik R. Funke, Khalid Daoudi, and Albert-Claude Boccara \\ Laboratoire d'Optique Physique, ESPCI, Université Paris VI, CNRS UPR 5, 10 Rue Vauquelin, \\ 75231 Paris, Cedex 05, France \\ Mickael Tanter and Mathias Fink \\ Laboratoire Ondes et Acoustique, ESPCI, Université Paris VII, CNRS UMR 7587, 10 Rue Vauquelin, \\ 75231 Paris, Cedex 05, France
}

(Received 5 February 2007; accepted 31 March 2007; published online 26 April 2007)

\begin{abstract}
The authors present a camera-based optical detection scheme designed to detect the transient motion created by the acoustic radiation force in soft elastic media. An optically diffusive tissue-mimicking phantom was illuminated with coherent laser light, and a high speed camera ( $2 \mathrm{kHz}$ frame rate) was used to acquire and cross-correlate consecutive speckle patterns. Time-resolved transient decorrelation of the optical speckle was measured as the result of localized motion induced by the radiation force and the associated propagating shear waves. The proposed technique is sensitive only to the low frequency transient motion induced in the medium by the radiation force. (C) 2007 American Institute of Physics. [DOI: 10.1063/1.2733604]
\end{abstract}

Optical imaging of biological tissue can provide relevant optical contrasts in terms of biomedical tissue characterization. ${ }^{1}$ However, the spatial resolution is limited for thick biological tissue because of the strong scattering. Recently, several techniques combining optics and ultrasound have been proposed to overcome this difficulty. ${ }^{2}$ Among these techniques, acousto-optic imaging is based on the detection of light modulated by ultrasound waves. ${ }^{3,4}$ As ultrasound can easily be focused in soft biological tissue, this method provides a way of discriminating the photons that travel along paths traversing the insonified region from the other photons. From the relative amount of modulated photons, optical information can therefore be derived with ultrasonic resolution. While the various methods differ in the type of detection involved (single detector, ${ }^{4,5}$ parallel multidetection of speckle fields, ${ }^{6-8}$ photorefractive crystal holography ${ }^{9,10}$ ), they aim at detecting the effect of ultrasonic oscillation, i.e., the effect of compressional waves, usually in the megahertz frequency range.

Meanwhile, in addition to purely oscillating compressive strain, high-intensity focused ultrasound may also generate low frequency strain in elastic media via the ultrasound radiation force. ${ }^{11}$ The radiation force corresponds to a transfer of momentum from the wave to a medium as the wave is either caused by reflected by impedance mismatches or attenuation. ${ }^{11}$ In the case of soft biological tissue, which attenuates ultrasound because of absorption and/or scattering by very weak random fluctuations in acoustic impedance, the radiation force density (unit force per unit volume) is given by the following formula: ${ }^{12}$

$$
f=\frac{2 \alpha I}{c},
$$

where $\alpha$ is the ultrasound attenuation coefficient (in Neper per unit distance), $I$ is the acoustic intensity (defined as the time-averaged ultrasound intensity over the ultrasound period), and $c$ is the speed of sound. In response to the radiation force, a soft-tissue-like medium is set into a motion that

a)Electronic mail: emmanuel.bossy@espci.fr involves time and amplitude scales very different from those of the compressive oscillatory strain due to the ultrasound wave. For focused radiation forces, the corresponding displacement fields have been described analytically in the literature. ${ }^{13,14}$ These displacements are essentially unipolar and have typical amplitudes of several micrometers occurring in about a millisecond. ${ }^{13,14}$ The motion is initiated predominantly at the ultrasound focus, and then propagates as a shear wave perpendicularly to the direction of the ultrasound. ${ }^{12,13,15}$ In the context of the interaction of light and ultrasound in optically diffusive media, a consequence of the radiation force has recently been discussed in a letter by Kim et al. ${ }^{16}$ These authors observed a $15 \%$ additional loss of speckle contrast generated by the acoustic radiation force, presented as a deleterious effect, in addition to the loss of contrast caused by the compressional wave itself through the acousto-optic effect. ${ }^{16}$

In this letter, we present a camera-based optical detection scheme designed to detect specifically the transient motion created by the radiation force in depth in an optically diffusive medium, while being insensitive to the acoustooptic modulation caused by the ultrasonic compressional waves. The proposed method aims at detecting the very small transient motion induced in a medium by the radiation force originating from local attenuation of ultrasound. This case corresponds to that of biological tissue, which presents no strong acoustic impedance mismatches. As the variation of the ultrasonic attenuation remains small in soft biological tissue, the amplitude of transient tissue motion is mainly related to the viscoelastic properties of the medium, i.e., the shear modulus and shear viscosity. ${ }^{14}$ Moreover, the proposed optical detection of this tissue motion involves the amount of photons that traversed the displaced region and therefore yields information on optical properties.

The experimental setup is shown in Fig. 1. A continuous $5 \mathrm{~W}$ frequency-doubled Nd:YAG laser source (Verdi, Coherent) was used to illuminate an optically diffusive tissuemimicking phantom. The phantom consisted of a $1 \%$ agar gel made optically diffusive by adding Intralipid. A concentration of $0.4 \%$ Intralipid $^{17}$ was used to obtain a reduced scattering coefficient of approximately $\mu \mathrm{s} \approx 6 \mathrm{~cm}^{-1}$. The di- 


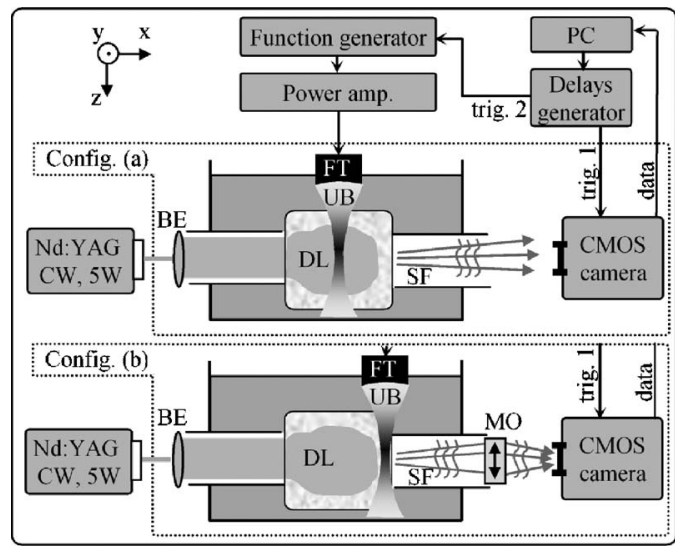

FIG. 1. Schematic diagram of the experimental setup. BE: beam expander, DL: diffuse light, UB: ultrasound beam, FT: focused transducer, SF: speckle field, MO: macro-objective.

mensions of the phantom were $40 \times 40 \times 40 \mathrm{~mm}^{3}$. A $5 \mathrm{MHz}$ spherically focused ultrasound transducer (A307-S, GE Panametrics) was used to focus high-intensity ultrasound bursts of millisecond duration in the phantom and generate transient displacements. A 1024 pixel $\times 1024$ pixel CMOS camera (HSS-4, Lavision) with a $2 \mathrm{kHz}$ frame rate was used to record the optical speckle pattern radiated by the sample. A tube $2 \mathrm{~cm}$ in diameter was used both to hold the phantom in the water and to collect light. A delay generator (BNC 565 , Berkeley Nucleonics) was used to trigger the camera acquisition (trigger 1) several milliseconds before triggering the ultrasound burst (trigger 2). Two configurations [(a) and (b)] of the setup were used, as shown on Fig. 1. In configuration (a), the ultrasound beam is located in the center of the phantom $(X=0 \mathrm{~mm})$, and the camera is used without optics to record the free-field speckle pattern at a distance of approximately $1 \mathrm{~m}$ from the phantom. In this case, the photons that traverse the ultrasound beam and reach the camera are multiply scattered before detection, and the camera records a speckle pattern with no spatial information. In configuration (b), the ultrasound focus is positioned on the output surface of the sample $(X=20 \mathrm{~mm})$. In this case, the photons affected by the ultrasound and reaching the camera are little affected by scattering. The camera's macro-objective was used to form a speckled image of the phantom surface on the detection plane of the camera and therefore to detect these photons with spatial information.

In this work, we show that it is possible to detect the low frequency motion induced in depth in the tissue phantom by the radiation force [Fig. 1, configuration (a)]. The configuration (b) of Fig. 1 is used to interpret the results obtained with configuration (a). For both configurations, the optical detection scheme consisted of spatial cross correlations between consecutive speckle patterns recorded with a $2 \mathrm{kHz}$ frame rate and a $0.5 \mathrm{~ms}$ exposure time. As transient nonoscillatory displacements generated by the ultrasonic radiation force change over a millisecond time scale, the high frame rate allows us to time-resolve the decorrelation created by those displacements while averaging out during the exposure time the modulation caused by purely ultrasonic oscillation at $5 \mathrm{MHz}$. In configuration (a), the spatial cross-correlation coefficient between consecutive pairs of entire speckle patterns was calculated as a function of time (using the corr2 function in MATLAB). This correlation coefficient quantifies
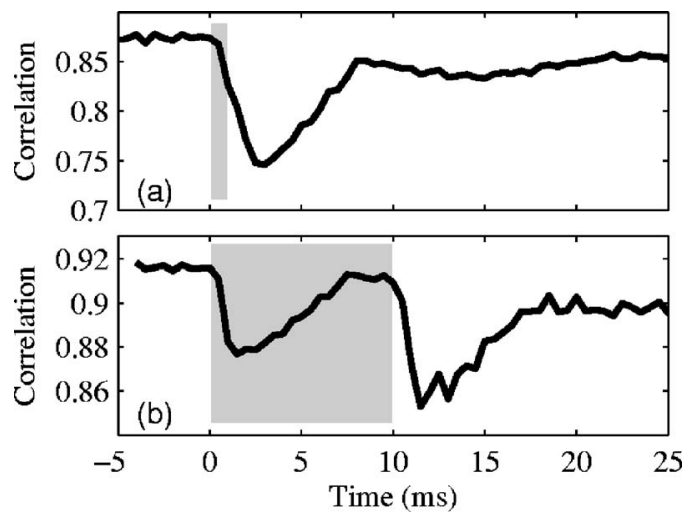

FIG. 2. Decorrelation curves measured with the experimental configuration (a) of Fig. 1 for a $1 \mathrm{~ms}$ ultrasound burst (a) and a $10 \mathrm{~ms}$ ultrasound burst (b) focused at depth in the tissue-mimicking phantom. The shaded regions indicate the position in time of the ultrasound bursts.

the resemblance between consecutive speckle patterns, and a decrease of the coefficient indicates a change from one image to the next. Configuration (b) was used in order to extract information on the motion at the surface from the recorded speckle images: cross-correlation images were obtained for each pair of images by calculating the cross-correlation coefficients between consecutive (in time) blocks of the speckle images subdivided into $64 \times 64$ blocks of $16 \times 16$ pixels $\left(272 \times 272 \mu \mathrm{m}^{2}\right)$.

Figure 2(a) plots the cross-correlation curve obtained for a $1 \mathrm{~ms}$ duration ultrasound burst. This curve clearly features a transient decorrelation, which lasts for several milliseconds. The fact that the decorrelation continues to grow after the ultrasound burst has been turned off proves that the detected effect is not caused by the ultrasonic oscillation of the compressional wave. Moreover, the duration of the decorrelation ( $\sim 7-8 \mathrm{~ms})$ suggests that the measurement is sensitive not only to the motion created at focus [which would yield a transient effect with typical response time of the order of $1 \mathrm{~ms}$ (Refs. 13 and 14)], but also to the associated shear waves propagating away from the focal region. ${ }^{12,13}$ Figure 2(b) plots the decorrelation measured for a $10 \mathrm{~ms}$ duration ultrasound burst, i.e., longer than the decorrelation duration observed in Fig. 2(a). The absence of decorrelation between 8 and $10 \mathrm{~ms}$, when the displacement has reached a steady state while the high-intensity ultrasound burst is still on, shows that the correlation factor is insensitive to the ultrasonic oscillation of the compressional wave. The second transient decorrelation pattern starting at $10 \mathrm{~ms}$ corresponds to the relaxation of the medium and the associated generation of shear waves after the radiation force has been turned off.

The same experiment was performed with configuration (b) of Fig. 1 in order to image the decorrelation created by the radiation force on the output surface. Figure 3 shows the

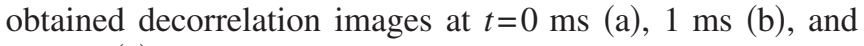
$1.5 \mathrm{~ms}$ (c) after the end of a $10 \mathrm{~ms}$ ultrasound burst. Figure 3(a) shows the decorrelation confined to the focal region during the first millisecond, and Figs. 3(b) and 3(c) show the decorrelation caused by the outgoing shear waves. The shear wave velocity derived in Fig. 3 is of the order of $1-2 \mathrm{~mm} / \mathrm{ms}$, in agreement with expected velocities for shear waves in soft-tissue-like media. ${ }^{12,13,15}$ The decorrelation curves obtained with configuration (a) therefore reflects the amount of transient motion generated by the radiation force, consisting of the initial displacement at focus followed 


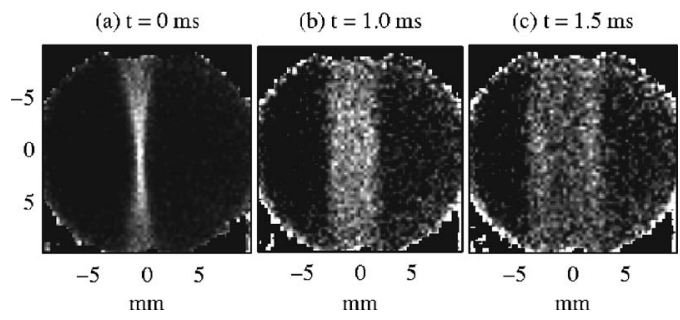

FIG. 3. Decorrelation images showing the motion generated by a $10 \mathrm{~ms}$ radiation force applied on the output surface of the sample, obtained with the experimental configuration (b) of Fig. 1. The images were taken at the end of the ultrasound burst (a), and after $1 \mathrm{~ms} \mathrm{(b)}$ and $1.5 \mathrm{~ms}(\mathrm{c})$.

by the propagation of shear waves. We infer that the decorrelation at a given time is directly related to the spatial integral of the displacement field. However, providing an analytical model relating the measured decorrelation to the displacement field is beyond the scope of this letter.

To illustrate the potential of the technique to detect optical and/or shear mechanical contrasts in depth in an optically diffusive medium, a phantom was made with a 10 $\times 10 \times 40 \mathrm{~mm}^{3}(X, Y$, and $Z$, respectively $)$ inclusion dyed with India ink, with mechanical properties identical to the rest of the phantom. An inclusion with a long dimension along the $Z$ axis was preferred to a smaller inclusion centered in the volume in order to avoid artificial impedance mismatch at the location of the push. Figure 4 plots the decorrelation curves measured in configuration (a) of Fig. 1 for three different locations of the focal region, in the middle of the inclusion $(X=0 \mathrm{~mm})$ and $10 \mathrm{~mm}$ away from the center of the inclusion $(X= \pm 10 \mathrm{~mm})$. The absence of decorrelation observed in the early part of the curve measured at $X=0 \mathrm{~mm}$ is a signature of the absorbing inclusion: as photons are absorbed by the dark inclusion, no decorrelation is expected in the speckle pattern as long as the displacement remains confined to the inclusion. Moreover, the decorrela-

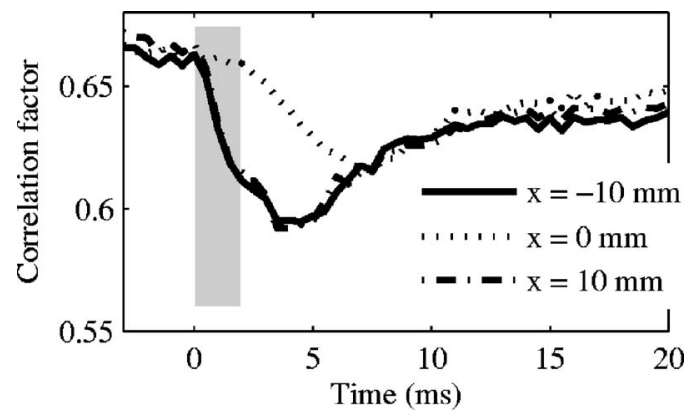

FIG. 4. Decorrelation curves measured for three different locations of the ultrasound focus in a phantom with a dark $10 \times 10 \times 40 \mathrm{~mm}^{3}$ inclusion centered at $X=0 \mathrm{~mm}$ (center of the phantom). The shaded region indicates the position in time of the $2 \mathrm{~ms}$ ultrasound burst. tion remains zero for about $2 \mathrm{~ms}$, which is in agreement with the time needed for the shear wave to exit the $1 \mathrm{~cm}$ wide dark inclusion at a velocity of about $2 \mathrm{~mm} / \mathrm{ms}$. As the later part of the decorrelation curve originates from shear waves propagation, its amplitude depends on the initial displacement at focus, as the source of the shear waves. In the experiment above, the three curves are identical after a few milliseconds, indicating that shear waves of identical amplitudes were launched at the three different locations of the push and therefore that the shear elastic properties were identical at these locations. Therefore, combining the amplitude of the late decorrelation curve with the initial decorrelation during the first millisecond provides a promising way to derive both optical and shear mechanical properties at the focus.

In conclusion, a technique for optoelastography was proposed based on the detection of time-resolved transient decorrelations of the optical speckle due to transient motion induced by a radiation force in an attenuating tissue-like medium. The observed decorrelation provides information about the motion initially generated at the focal region of the ultrasound beam and on the associated generated shear wave. The technique provides millimetric lateral resolution as the outgoing shear waves do not exit the focal region before typically $1 \mathrm{~ms}$. The decorrelation curves in configuration (a) contain information about the amount of photons in the displaced region (optical information) as well as information about the amplitude of the displacement (shear mechanical properties).

${ }^{1}$ V. V. Tuchin, Handbook of Optical Biomedical Diagnostics (SPIE, Washington, 2002).

${ }^{2}$ L. V. Wang, Dis. Markers 19, 123 (2003).

${ }^{3}$ W. Leutz and G. Maret, Physica B 204, 14 (1995).

${ }^{4}$ L. H. Wang, S. L. Jacques, and X. M. Zhao, Opt. Lett. 20, 629 (1995).

${ }^{5}$ A. Lev, Z. Kotler, and B. G. Sfez, Opt. Lett. 25, 378 (2000).

${ }^{6}$ S. Leveque, A. C. Boccara, M. Lebec, and H. Saint-Jalmes, Opt. Lett. 24, 181 (1999).

${ }^{7}$ G. Yao, S. L. Jiao, and L. V. Wang, Opt. Lett. 25, 734 (2000).

${ }^{8}$ M. Gross, P. Goy, and M. Al-Koussa, Opt. Lett. 28, 2482 (2003).

${ }^{9}$ T. W. Murray, L. Sui, G. Maguluri, R. A. Roy, A. Nieva, F. Blonigen, and C. A. DiMarzio, Opt. Lett. 29, 2509 (2004).

${ }^{10}$ F. Ramaz, B. C. Forget, M. Atlan, A. C. Boccara, M. Gross, P. Delaye, and G. Roosen, Opt. Express 12, 5469 (2004).

${ }^{11}$ G. R. Torr, Am. J. Phys. 52, 402 (1984).

${ }^{12}$ J. Bercoff, M. Tanter, and M. Fink, IEEE Trans. Ultrason. Ferroelectr. Freq. Control 51, 396 (2004).

${ }^{13}$ A. P. Sarvazyan, O. V. Rudenko, S. D. Swanson, J. B. Fowlkes, and S. Y. Emelianov, Ultrasound Med. Biol. 24, 1419 (1998).

${ }^{14} \mathrm{~J}$. Bercoff, M. Tanter, M. Muller, and M. Fink, IEEE Trans. Ultrason. Ferroelectr. Freq. Control 51, 1523 (2004).

${ }^{15}$ J. Bercoff, M. Tanter, and M. Fink, Appl. Phys. Lett. 84, 2202 (2004).

${ }^{16}$ C. Kim, R. J. Zemp, and L. H. V. Wang, Opt. Lett. 31, 2423 (2006).

${ }^{17}$ H. J. Van Staveren, C. J. M. Moes, J. Vanmarle, S. A. Prahl, and M. J. C. Vangemert, Appl. Opt. 30, 4507 (1991). 